\title{
Non-invasive diagnosis of portal vein occlusion by radionuclide angiography
}

\author{
P MacMathuna, M K O’Connor, D G Weir, P W N Keeling
}

\begin{abstract}
The accuracy of non-invasive radionuclide angiography in detecting portal vein occlusion was assessed in 61 patients -10 with portal vein occlusion confirmed by conventional portography, 25 with chronic liver disease and a patent portal vein (mild $=12$, severe $=13$ ), and 26 with normal liver function, who served as controls. The median percentage portal venous flow for the portal vein occlusion group was $8 \%$ (range 1-30) (consistent with negligible flow) compared with 78\% (52-87) for control subjects $(\mathrm{p}<0.005)$ and $68 \%(61-80)$ and $49 \%$ (23-59) respectively for patients with mild and severe liver disease $(p<0.001$ and $p<0.005)$. At a portal venous inflow of $<20 \%$, the proceedure had a specificity of $100 \%$ and sensitivity of $90 \%$ in diagnosing portal vein occlusion. Non-invasive radionuclide angiography provides a safe and accurate screening method for evaluating portal vein patency or occlusion in the investigation of portal hypertension or before liver transplantation.
\end{abstract}

(Gut 1992; 33: 1671-1674)

Complete or partial occlusion of the portal vein is a common cause of portal hypertension in childhood. ${ }^{12}$ In adults, portal vein occlusion (PVO) may complicate chronic liver disease but more frequently results from intra-abdominal sepsis, neoplasia, or a thrombotic disorder. ${ }^{3}$ In many cases, however, no aetiological factor is found. Gastrointestinal haemorrhage is the commonest clinical presentation, while the presence of ascites and encephalopathy usually indicate associated liver dysfunction. Early recognition of the condition is important since management may differ from that of cirrhosis, particularly if portacaval surgery is considered. ${ }^{3}$ PVO is a contraindication to liver transplantation and after transplantation, thrombosis of the portal vein (or hepatic artery) is a recognised cause of graft dysfunction. ${ }^{+}$Consequently, defining the patency or occlusion of the portal venous system is equally important in pretransplant assessment
TABLE I Clinical details of the three patient groups

\begin{tabular}{llll}
\hline & PVO & Cirrhosis & Control $^{\star}$ \\
\hline No & 10 & 25 & 26 \\
Mean age (years) & 47 & 52 & 54 \\
Male/female & $5 / 5$ & $10 / 15$ & $14 / 12$ \\
Liver disease & $3 / 10$ & $25 / 25$ & $0 / 26$ \\
\hline
\end{tabular}

$\mathrm{PVO}=$ portal vein occlusion

${ }^{\star}$ Controls = patients undergoing bone, cardiac, or cerebra scanning.

and in post-transplant graft dysfunction.

At present, the accurate diagnosis of PVO requires either arterial portography or splenoportography, both of which are invasive radiological proceedures that may have complications, particularly in patients with a coagulopathy. ${ }^{56} \mathrm{~A}$ reliable non-invasive diagnostic technique would represent a considerable advance. Recent reports have advocated either ultrasonography ${ }^{78}$ or computer tomography. ${ }^{9}$ The application of radionuclide angiography in assessing portal vein patency or occlusion has received limited attention. We have previously described a new non-invasive dynamic hepatic scintigraphic technique to define the portal venous and hepatic arterial contributions to total liver perfusion. ${ }^{10}$ More recent in vivo animal studies provide experimental evidence to validate this technique." Preliminary use of this method in a small number of patients successfully detected PVO. ${ }^{10}$ The present study aimed to assess the accuracy of this technique in the diagnosis of PVO in a larger cohort of patients.

\section{Patients and methods}

PATIENTS

Three groups of patients were included in this investigation (Table I).

\section{Group 1: patients with PVO (Table II)}

Ten patients ( 5 men and 5 women) with a mean age of 47 years (range 21-72) were assessed. The diagnosis of PVO was confirmed in each case by

TABLE II Individual clinical and radiological details concerning the 10 patients with portal vein occlusion

\begin{tabular}{|c|c|c|c|c|c|c|}
\hline $\begin{array}{l}\text { Patient } \\
\text { no }\end{array}$ & $\begin{array}{l}\text { Age } \\
\text { (years) }\end{array}$ & Aetiology & Cirrhosis & Clinical features & Diagnostic method & $\begin{array}{l}\text { Caviernous } \\
\text { transformation }\end{array}$ \\
\hline 1 & 54 & Idiopathic & No & Oesophageal varices & Angiography & No \\
\hline 2 & 21 & Idiopathic & No & Oesophageal varices & Angiography & No \\
\hline 3 & 55 & Pancreatitis & No & Oesophageal varices & Angiography & No \\
\hline 4 & 35 & Idiopathic/? trauma & Yes & $\begin{array}{l}\text { Oesophageal varices/hepatic } \\
\text { encephalopathy }\end{array}$ & Angiography & No \\
\hline 5 & 63 & Cirrhosis & Yes & $\begin{array}{l}\text { Oesophageal varices/hepatic } \\
\text { encephalopathy }\end{array}$ & Angiography & No \\
\hline 6 & 40 & Polycylhaemia rubra vera & No & Oesophageal varices/ascites & Angiography & No \\
\hline 7 & 69 & Idiopathic & No & Oesophageal varices & Angiography & No \\
\hline 8 & 38 & Idiopathic & No & Asymptomatic & Laparotomy+angiography & Yes \\
\hline 9 & 72 & $\begin{array}{l}\text { Cirrhosis/hepatocellular } \\
\text { carcinoma? }\end{array}$ & Yes & Oesophageal varices & Angio + post mortem & Yes \\
\hline 10 & 51 & Idiopathic & No & Oesophageal varices & Angiography & Yes \\
\hline
\end{tabular}




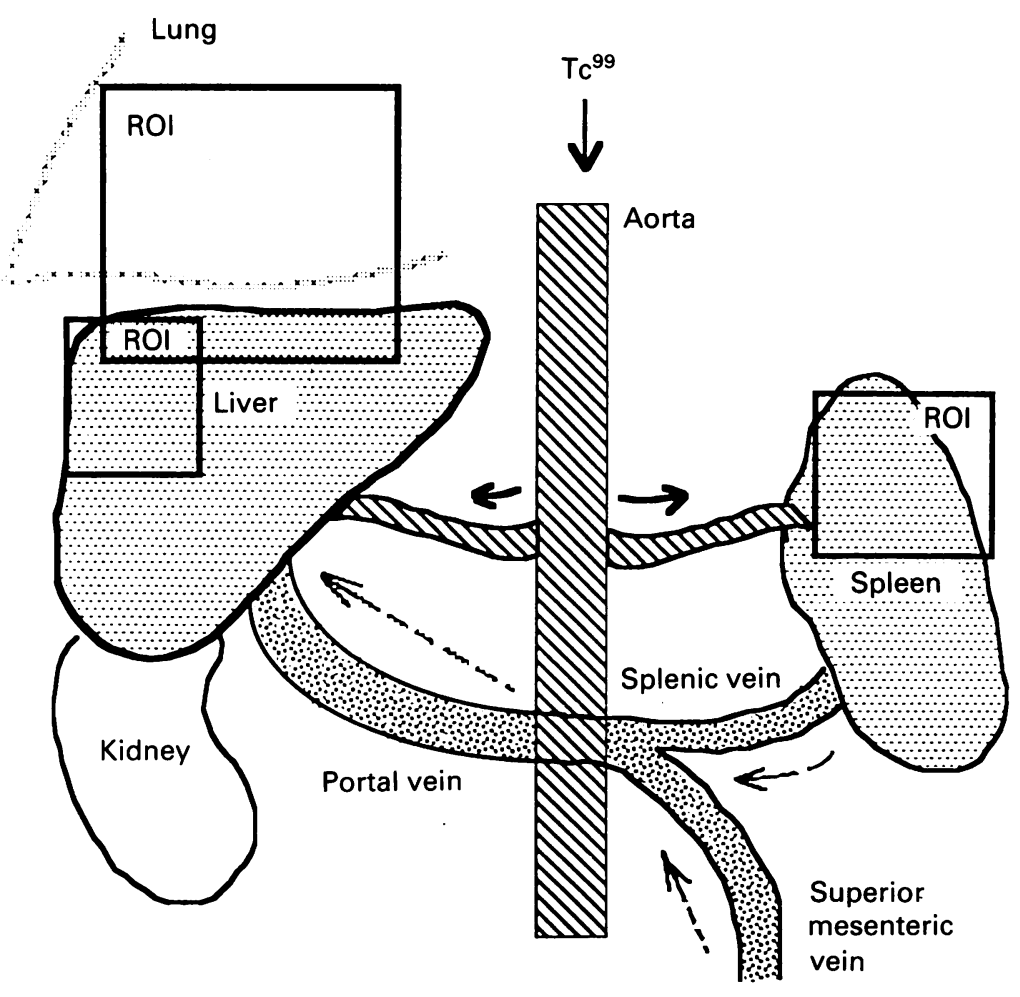

Figure 1: Schematic outline illustrating the field of view of the gamma camera including regions of interest (ROIs) over the liver, spleen, lung, and left kidney. conventional arterial portography or splenoportography with additional conclusive evidence from laparotomy and post mortem examination in two cases (see Table II). In three patients there was coexisting liver disease and in one of these patients (patient 9), the PVO was associated with the development of hepatocellular carcinoma. Pancreatitis (patient 3) and polycythaemia rubra vera (PRV) (patient 6), accounted for two other cases while no cause was evident in the remaining five patients (idiopathic).

\section{Group 2: patients with chronic liver disease without PVO}

This group comprised 25 patients ( 12 men and 13 women) with a mean age of 51 years (range 30-74), all of whom had documented chronic liver disease (clinical, biochemical, and histological criteria) with patent portal vein as shown radiologically. The aetiological factors for the liver disease included alcohol $(n=19)$, chronic active hepatitis $(n=3)$, Wilson's disease $(n=2)$, and haemochromatosis $(n=1)$. Twenty of the patients had portal hypertension, shown by the presence of endoscopically proved oesophageal varices, with or without splenomegaly. The patients were classified according to the severity of liver disease using the modified Child's-Pugh score $^{12}$ into two groups: mild disease with a Child-Pugh score of $<8 \quad(n=12)$ and severe disease with a score of $>8(n=13)$.

Group 3: normal control subjects

There were 26 control subjects ( 14 men and 12 women) with a mean age of 54 years (range 2578) none of whom had any clinical, biochemical, or radiological evidence of liver disease or extrahepatic portal hypertension. These patients were undergoing cardiac, bone, or cerebral isotope scanning and dynamic liver scintigraphy could be incorporated without unnecessary additional exposure to radionuclides.

Written informed consent was obtained from each patient before the study.

\section{METHODS}

The technique of dynamic hepatic radionuclide angiography has been described previously in detail. ${ }^{10}$ In brief, fasting patients were positioned supine beneath a large field of view gamma camera (General Electric, Maxi II). After a 5 minute resting period, in patients with $\mathrm{PVO}$ or liver disease, $370 \mathrm{MBq}(10 \mathrm{mCi})$ of ${ }^{99 \mathrm{~m}} \mathrm{Tc}$ pertechnetate were administered by bolus peripheral intravenous injection followed rapidly by a $30 \mathrm{ml}$ saline flush to ensure rapid transit of the radionuclide into the heart. In control subjects, 555-740 MBq (15-20 mCi) of ${ }^{99 m} \mathrm{Tc}$ labelled human albumin, methylene diphosphonate, or glucoheptonate were administered in a similar manner. Computer image acquisition began at the onset of the injection. Images were acquired onto a $64 \times 64$ word matrix at a rate of 0.5 seconds/image for 100 seconds and stored on an $\mathrm{A}^{2}$ computer system for subsequent analysis (Medical Data Systems, Ann Arbor, MI, USA). The procedure takes approximately 3 minutes to complete.

Preliminary data analysis involved a summation of the initial 30 images to form a composite image outlining the arterial phase of tracer transit through the hepatobiliary system. Regions of interest (ROIs) were drawn over the liver, spleen, lungs, and left kidney ensuring that the liver ROI excluded the descending aorta and right kidney (Fig 1). The ROIs were then used to generate time-activity curves (TACs). Because of interference from the right lung into the liver ROI, a correction factor was applied to take account of this 'cross-talk', resulting in a corrected liver TAC (Fig 1). Deconvolution analysis of the TACs was used to improve separation of the arterial and portal venous
Figure 2: Left: corrected liver and spleen curves. Centre: curves following deconvolution analysis. Right: magnitude of the spleen modified so that the upleen modified so that the
upslope matches that of the liver curve. $A_{\mathrm{S}}$ and $A_{\mathrm{L}}$ represent the areas under the spleen and liver curves respectively.
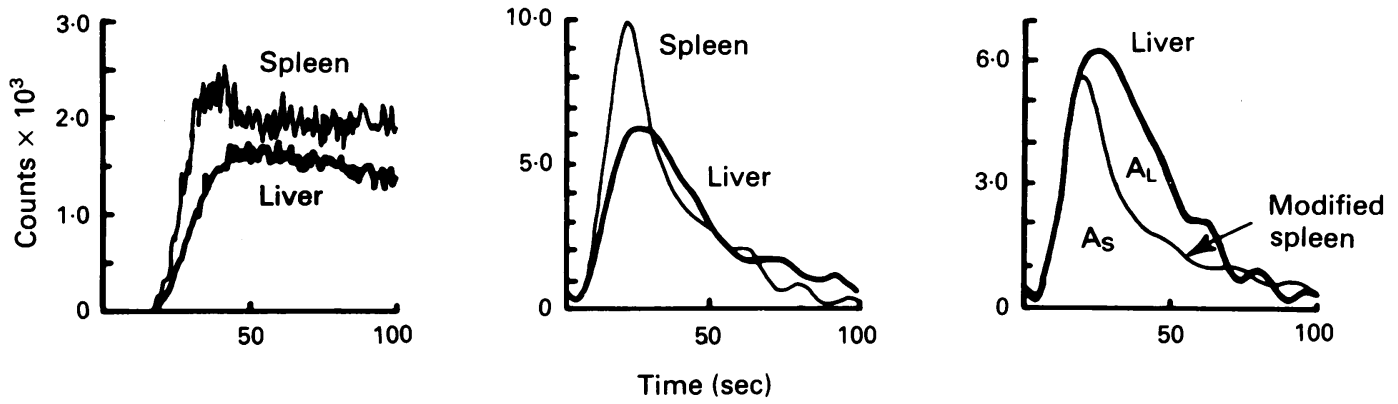
phases of total liver blood flow. In addition, this method takes account of tracer recirculation and therefore allows analysis of the deconvoluted curves by area under the curve as a measure of blood flow. The liver, spleen, and left kidney TACs were deconvoluted with the lung curve using a modified fast Fourier transform technique as described by Juni et al. ${ }^{13}$ If the assumption is made on an anatomical basis, that blood flow through the splenic artery has a similar pattern to hepatic arterial flow, then the splenic TAC can be used as a model for hepatic arterial flow. In order to compensate for differences in absolute flow and in attenuation by overlying tissues, the splenic TAC was multiplied by a constant so that the upslope of the splenic TAC overlies the early segment of the total liver TAC (Fig 2). Thus, the modified splenic TAC approximates to the hepatic arterial TAC. The liver and modified splenic TAC are then integrated to yield the areas under the curve, $A_{L}$ and $A_{S}$, respectively (Fig 2). The hepatic arterial component (\% HA) was calculated from the formula:

$$
\% \mathrm{HA}=\mathrm{A}_{\mathrm{S}} / \mathrm{A}_{\mathrm{L}} \times 100
$$

and the portal venous component (\% PV) determined as:

$$
\% \mathrm{PV}=100-\% \mathrm{HA} \text {. }
$$

Recent in vivo experimental animal studies have validated this deconvolutional technique to define \% HA and \% PV by dynamic radiouclide angiography. ${ }^{11}$

The results are expressed as \% $\mathrm{HA}$ and \% PV and are shown as median (range). Specificity and sensitivity values were calculated and statistical analysis was performed using the MannWhitney $U$ test for non-parametric data. $p<0.05$ was taken as the level of significance.

Figure 3: Results of the calculated percentage portal venous flow for control subjects, patients with mild and severe liver disease, and patients with portal vein occlusion $(P V O)$. The cross bars represent medians.

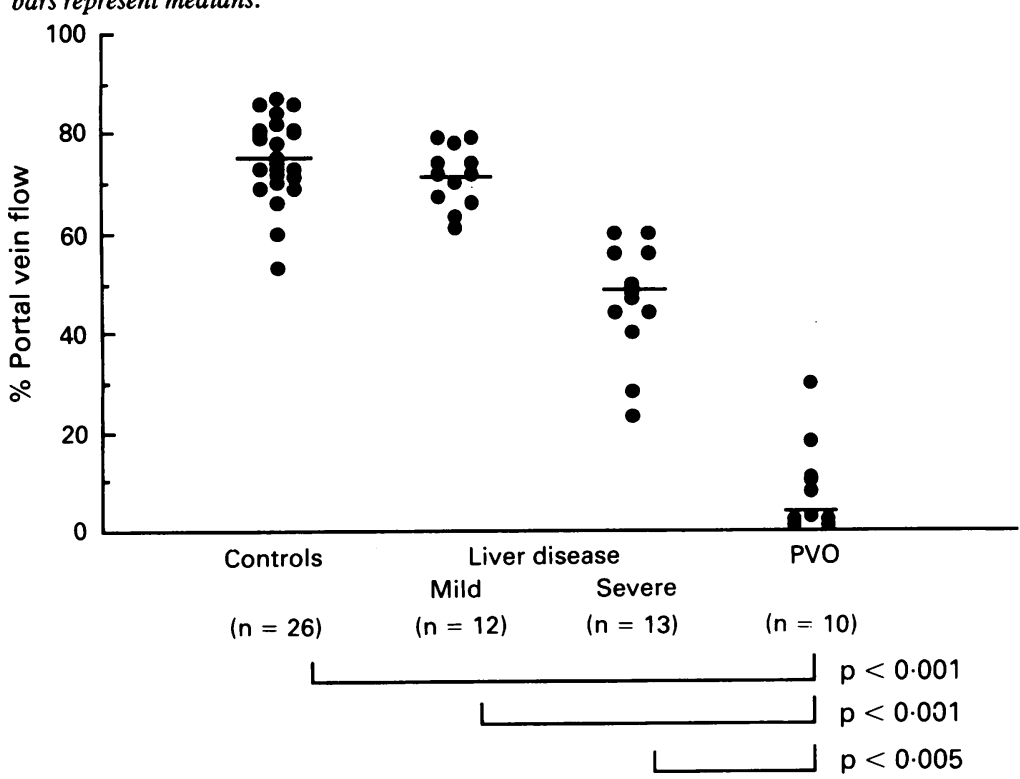

\section{Results} flow and \% PV flow in each of the 10 patients with PVO (group 1). The \% PV flow in group 1 was $8.6 \%$ (range 1-30), which reflects negliable portal venous inflow to the liver. When the three
TABLE III Individual results of the hepatic arterial (\% HA) and portal venous (\% PV) flow for the 10 patients with portal venous occlusion

\begin{tabular}{llc}
\hline Patient no & $\% \mathrm{HA}$ & $\% \mathrm{PV}$ \\
\hline 1 & 98 & 2 \\
2 & 92 & 8 \\
3 & 98 & 2 \\
4 & 97 & 3 \\
5 & 99 & 1 \\
6 & 90 & 10 \\
7 & 99 & 1 \\
Mean (patients 1-7) & $96 \cdot 4$ & $3 \cdot 6$ \\
$8^{\star}$ & 70 & 30 \\
$9^{\star}$ & 89 & 11 \\
$1^{\star}$ & 82 & 18 \\
Mean (patients 1-10) & $91 \cdot 4$ & $8 \cdot 6$ \\
\hline
\end{tabular}

*These patients also had cavernous transformation of the portal vein.

patients with coexisting cavernous transformation of the portal vein were excluded, the calculated \% PV was further reduced to 3\% (range 1-10). Only the three patients with cavernous transformation had a \% PV flow of more than $10 \%(11 \%, 18 \%$, and $30 \%$ respectively) (Table III).

Figure 3 illustrates the differences in \% PV flow between the patients with PVO and the two control groups (2 and 3). The \% PV flow for control subjects (group 3) was $78 \%$ (range, 5386) which is consistent with known physiological estimates. ${ }^{1+}$ In group 2, a progressive reduction in the \% PV contribution with increasing severity of liver disease was observed, from $68 \%$ in patients with mild disease (range 61-80) ( $>0.10 v$ controls) to $49 \%$ in the group with severe disease (range 22-61) $(\mathrm{p}<0.001 v$ controls). In two patients with decompensated cirrhosis (Child-Pugh score $\mathrm{C}_{12 \& 13}$ ), the \% PV flow was $23 \%$ and $28 \%$, respectively. The $\%$ PV flow was significantly reduced in the PVO group when compared with both control groups 2 and 3 $(\mathrm{p}<0.0001 v$ controls, $\mathrm{p}<0.001 v$ mild, and $\mathrm{p}<0.005 v$ severe liver disease (Fig 3)).

For a \% PV flow of $20 \%$ or less, the specificity and sensitivity of this proceedure in accurately

Table III outlines the individual values for $\% \mathrm{HA}$ detecting PVO were $100 \%$ and $90 \%$, respectively. At a $\%$ PV flow of $30 \%$ or less, the specificity was reduced to $85 \%$ while the sensitivity was increased to $100 \%$

\section{Discussion}

Confirmation of portal vein patency or occlusion relies predominantly upon venous phase arterial portography or splenoportography, both invasive and time consuming radiological procedures that may have complications. Several investigators have attempted to define the portal venous and hepatic arterial contributions to total liver blood flow by non-invasive means including ultrasound ${ }^{78}$ and radionuclide angiography. ${ }^{10}{ }^{15-18}$ The degree of portasystemic shunting in cirrhosis has also been examined by scintigraphy, however per rectal administration of the radionuclide is required. ${ }^{2021}$ The present investigation is the first report of radionuclide angiography specifically used to assess the patency/occlusion of the portal venous system.

We have shown that the \% PV flow in all the patients with PVO was $8.6 \%$ with a range of 1 to $30 \%$, indicating that blood flow to the liver may become almost entirely dependant upon the 
hepatic artery. Furthermore, when the patients with cavernous transformation are excluded, the observed portal venous input to the liver becomes negligible $(<3 \%)$. Defining occlusion of the portal vein as a $\%$ PV flow of $20 \%$ or less, the technique is highly specific and sensitive. The results indicate therefore that this radionuclide technique accurately detects occlusion to portal venous inflow to the liver, particularly in patients without coexisting cavernous transformation. Another report also demonstrated the absence of any significent portal venous flow in two patients in whom portal vein thrombosis was subsequently confirmed at necropsy. ${ }^{16}$

There are two circumstances that may potentially reduce the diagnostic accuracy of this technique. Firstly, the presence of a cavernous transformation or 'cavernoma' represents the development of multiple periportal collateral channels in order to bypass the obstruction within the portal vein. Consequently, this provides a potential route for portal venous blood to reach the liver. The \% PV flow observed in the three patients with cavernous transformation included in the present study ranged from $11 \%$ up to $30 \%$. In these circumstances, the sensitivity of this method of detecting portal vein occlusion may be diminished. Nevertheless, only one patient with documented PVO in the present study had a \% PV flow of greater than $20 \%$.

Secondly, a major reduction in portal venous inflow to the liver associated with reversed (caudal) blood flow, may occur in the absence of obstruction within the portal venous system, ${ }^{22}$ as observed in two of the patients with decompensated alcoholic cirrhosis with \% PV flows of $28 \%$ and $23 \%$ respectively (Fig 3). Consequently, \% PV flow values of between 20 and $30 \%$ fall within what might be termed a diagnostic 'grey area'. It seems appropriate at the present time, therefore, that the suspicion of PVO, particularly in patients with decompensated liver disease as indicated by the presence of an appreciably reduced portal blood flow, needs to be confirmed by conventional arterial portography. Nevertheless, at a $\%$ PV flow of below $20 \%$, radionuclide angiography allowed a clear distinction to be made between patients with a patent and obstructed portal venous system.

In conclusion, this study shows that noninvasive radionuclide angiography is of benefit in screening for obstruction within the portal vein as part of the preliminary investigation of portal hypertension and chronic liver disease or during the assessment for liver transplantation. This method may also be helpful in confirming the patency/occlusion of a portacaval shunt. The proceedure is safe and inexpensive. Further evaluation of radionuclide angiography in comparison with other non-invasive methods (ultrasound, computed tomography, and nuclear magnetic resonance) and conventional arterial portography merits consideration.

Preliminary results of this study were presented to British Society Preliminary results of this study were presented to British Society of Gastroenterology meeting, Septem
abstract form in Gut 1988; 29: A1461.

1 Webb LJ, Sherlock S. The aetiology, presentation and natural history of extrahepatic portal venous obstruction. $Q 7 \mathrm{Med}$ 1979; 48: 627-39.

2 Alvarez F, Bernard O, Brunelle F, et al. Portal obstruction in children 1: clinical investigation and haemorrhage risk. f Pediatr 1983; 103: 696-702.

3 Triger DR. Extrahepatic portal venous obstruction. Gut 1987 ; 28: 1193-7.

4 Dixon A, Sherwood T. Radiological assessment. In: Calne R ed. Liver transplantation: The Cambridge-King's College Hospital experience. London: Grune \& Stratton, 1987: 91-3. 5 Bergstrand I. In: Abrams B, ed. Angiology. Boston, Mass: 1971; ii: 1030.

6 Burcharth F. Safe splenoportography: objective confirmation is still required. Hepatology 1988; 8: 425-6.

7 Raby N, Karani J, Powell-Jackson P, Meire H, Williams R. Assessment of portal vein patency: comparison of arterial portography and ultrasound scanning. Clin Radiol $1988 ; 39$. 381-5.

8 Webb LJ, Berger LA, Sherlock S. Grey-scale ultrasonography of portal vein. Lancet 1977; ii: 675-7.

9 Mathieu D, Vasile N, Grenier P. Portal thrombosis: dynamic CT features and course. Radiology 1984; 154: 737-41.

10 O'Connor MK, MacMathuna P, Keeling PWN. Hepatic arterial and portal venous components of liver blood flow: dynamic scintigraphic study. F Nucl Med 1988; 29: 466-72.

11 O'Connor MK, Krom RF, Carton EG, et al. Ratio of hepatic arterial to portal venous blood flow - validation of radionuclide techniques in an animal model. $F$ Nucl Med 1992; 33 239-45.

12 Pugh RNH, Murray-Lyon IM, Dawson JL, Pietroni MC, Williams R. Transection of the oesophagus for bleedin oesophageal varices. Br $\mathcal{F}$ Surg 1973; 60: 646-9.

13 Juni JE, Thrall JH, Froelich J, Hickwa RD, Clinthorne NH A simple technique for reducing deconvolutional artifact in A simple technique for reducing deconvolutional

14 Campra JL, Reynolds TB. The hepatic circulation. In: Arias I Popper H, eds. The Liver: biology and pathobiology. New Popper H, eds. The Liver: biology

15 Biersack HJ, Torres J, Thelan M, Monzon O, Winkler C. Determination of liver and spleen perfusion by quantitative sequential scintigraphy: results in normal subjects and patients with portal hypertension. Clin Nucl Med 1981; 6 : 218-20.

16 Wraight EP, Barber RW, Ritson A. Relative hepatic arteria and portal flow in liver scintigraphy. Nucl Med Commun 1982; 3: 273-9.

17 Fleming JS, Ackery DM, Walmsley BH, Karran SJ. Scintigraphy of arterial and portal blood supplies to the liver. $\dddot{f}$ Nucl Med 1983; 24: 1108-13.

18 Parkin A, Robinson PJ, Baxter P, Leveson SH, Wiggins PA Giles GR. Liver perfusion scintigraphy - method, normal Giles GR. Liver perfusion scintigraphy - method, normal range and laparotomy corre

19 Sarpar R, Tarcan YA. An improved method of estimating the portal venous fraction of total hepatic blood flow from computerised radionuclide angiography. Radiology 1983; 147: $559-62$.

20 Yen CK, Koblik P, Breznock B, et al. Portasystemic shun fraction quantification using transrectal administration of iodine-123 iodoamphetamine in dogs with chronic bile duct ligation and after propranolol administration. $7 \mathrm{Nucl} \mathrm{Med}$ 1989; 30: 1702-7.

21 Kashiwagi T, Azuma M, Ikawa T, et al. Portasystemic shunting in portal hypertension: evaluation with portal scintigraphy using transrectal administered I-123 IMP Radiology 1988; 169: 134-40.

22 Rector WG, Hoefs JC, Hossack KF, Everson GT. Hepatofugal portal flow in cirrhosis: observations on hepatic haemodynamics and the nature of the arterioportal communications. Hepatology 1988; 8: 16-20. 